\title{
QUELQUES FIGURES MAGIQUES DES BATAKS ET LEUR ORIGINE
}

\author{
Contribution à la science de la magie des Bataks
}

Pat Gerard Louwrens Tichelman

Avec 8 illustrations

Le Batak connaît un nombre considérable de signes de défense ou de signes protecteurs. L'art de se rendre invulnérable (hobal: Sim. bat.; parhobolon: Toba batak) forme une partie de la science des prêtres (Datoe). Parmi les radjahs (Sim. bat.) ou signes de défense dont les datoes ont coutume de se servir, on trouve toutes sortes de figures dessinées sur divers objets, entre autres des ustensiles d'usage courant et des instruments dont on se sert lors de cérémonies au cours desquelles on prononce des prières, des sentences arbitraires et des formules magiques ( $\mathrm{tab}$ as, mintora $=$ mantra: scr.). On considère que ces figures ont un effet protecteur contre le pouvoir nuisible des esprits malins. Elles sont alors purement passives; mais elles peuvent aussi neutraliser des influences magiques néfastes et même être utilisées activement pour se venger ou pour renvoyer à des adversaires le mal imaginé par eux (pangoelak: Sim. bat.). La fig. 1, I, représente une des plus importantes de ces figures, le bindoe matoga (bindoe matogoeh: Sim. bat.), qui est formé de deux carrés entremêlés, dont les angles sont terminés par des boucles. Le carré extérieur est parfois appelé bindoe matoga, le carré intérieur bindoe matogoeh. Matoga veut dire: "posséder un pouvoir sur lequel on peut compter»; matogoeh signifie: "fort par parenté, être sûr». Un grand nombre de cérémonies magiques a lieu sur, autour et à l'intérieur de cette figure tracée sur le sol (fig. 2). La personne qui se trouve à l'intérieur est protégée contre les esprits mauvais, comme si elle se trouvait dans une forteresse. Les figures 1 , II, et 1 , III, représentent le sceau de Salomon sous ses formes féminine et masculine (tapak Radjah Soeleman betina et djantan: mal.; tapak Radjah Soeleman naboroe et dalahi: Sim. bat. ${ }^{1}$ ).

La légende batake concernant l'origine de cette figure et répandue dans le domaine de Timosr ou Simaloengoen, relate que le roi Salomon (Soeleman) avait proclamé dans son royaume une grande fête de propitiation, parce que beaucoup le jalousaient. Non seulement les hommes, mais aussi les animaux de la forêt, les reptiles et les oiseaux étaient mal intentionnés à son égard, à cause de sa grande richesse et de son sens extraordinaire de la justice. Il appliquait rigoureusement, en effet, les prescriptions de l'adat, spécialement en ce qui concerne la parenté et la moralité (adat partoetoeran: Sim. bat. et Toba bat.), le pouvoir (adat hamoeliaon: Sim. bat. et Toba bat.) et le droit (adat habonaran: Sim. bat.). Le roi Salomon pria le Tout-Puissant de lui prêter secours. Le Tout-Puissant lui envoya un ange nommé Nabi Noehoem, qui, d'après certains Bataks, serait Nahoum, un des 12 "petits prophètes ${ }^{2}$. Celui-ci lui révèla des figures magiques (radjah-radjah: Sim. bat.) qui étaient des moyens de protection contre les puissances diaboliques.

${ }^{1}$ Tapak veut dire impression ou trace de pied ou de main, tapak-tapak: plante du pied ou paume.

${ }^{2}$ Cette identification est très improbable, vu que le prophète $\mathrm{Nahoem}$ ne joue aucun rôle dans la tradition musulmane, et que l'on ne peut voir une influence chrétienne dans cette légende. On pourrait penser à un abâtardissement de Noeh $=$ Noé, mais il semble encore plus plausible que Noehoem équivaut au Noen arabe (avec "ou» long), soit poisson, et Nabi Noehoen = Dhoel-Noen = l'Homme du poisson, c'est-à-dire le prophète Jonas. 


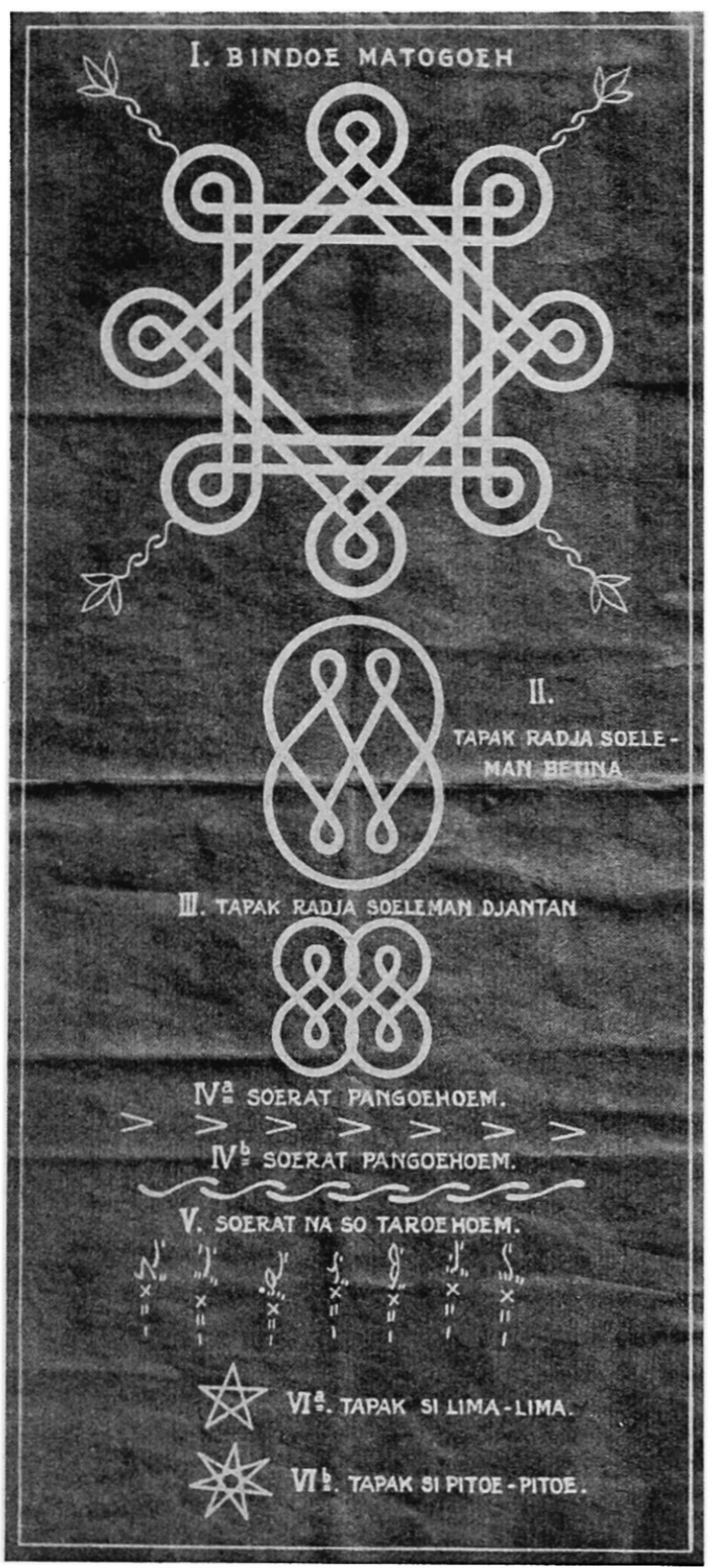

Fig. 1, I - VIb. Choix de quelques signes magiques des Bataks les plus usités. Photo G. L. Tichelman.

Dans toutes les influences qui l'entourent et peuvent lui être néfastes, l'homme primitif voit l'œuv re de forces surnaturelles, mal intentionnées à son égard. Le monde est rempli de puissances qu'il se représente comme étant des démons menaçant son bonheur et son existence, qui exercent une pression sur sa vie et qui le poursuivent du berceau jusqu'au tombeau. Les bons esprits veulent aussi être traités avec des égards. Leur mauvaise humeur est vite éveillée et ils punissent inexorablement envoyant maladies, mort et calamités, même lorsqu'on est involontairement en défaut. Aussi le Batak croit-il bien faire en prenant le certain pour l'incertain. Les signes magiques révélés au Radjah Soeleman possédaient le pouvoir bienfaisant de détourner de manière efficace toutes ces influences mystérieuses nuisibles, aux effets incalculables. Le Sigillum Salomonis, symbole du Logos, aussi nommé étoile de David, est un signe juif très ancien, composé de deux triangles équilatéraux, glissés l'un dans l'autre. Si la pointe est braquée vers le haut, le triangle, dans ce cas symbole du feu, représente le principe créateur suprême, tandis qu'il faut imaginer les deux extrémités de la base comme étant l'esprit et la matière dans lesquels le principe suprême se révèle. Lorsque la pointe est tournée vers le bas, dans ce cas symbole de l'eau, il faut y reconnaître l'homme, le fils, comme produit de l'esprit et de la matière. Glissés l'un dans l'autre et ne formant qu'une figure, les triangles représentent le principe premier masculin, donnant la chaleur et la fécondation, et le principe premier féminin, celui de la conception, tandis que le principe de la Trinité y trouve également son expression sous la forme "révélée» et "non révélée». H. A. WINKLER ${ }^{3}$ dit que le pentagramme et l'hexagramme servent indifféremment comme sceau de Salomon. Un cercle, à considérer comme la lettre ha' arabe (la 27e lettre de l'alphabet arabe, qui est placée au commencement ou à la fin d'un alinéa), semble être originellement le premier dans la série des "sept sceaux». Or, la valeur numérique de ha' est 5, ce qui explique le remplacement par pentagramme (et par le

${ }^{3}$ H. A. WINkLER: Siegel und Charaktere in der mohammedanischen Zauberei. Studien zur Geschichte und Kultur des islamischen Orients, 7, Leipzig 1930. 
synonyme hexagramme). "Vielleicht schon unter dem Einfluß des eindringenden Pentaund Hexagrammes wurde dieses Ha' verdoppelt (G. Tichelman, Cultureel Indie 1, 1939 p. 330)." La signification originale de ha' est huwa, comme nom de Dieu*.

La figure du tapak $\mathrm{R}$ adjah Soeleman des Bataks ne concorde donc pas avec ce qui est ordinairement considéré comme le sceau de Salomon. Il s'agit en effet de ce symbole stylisé: les deux triangles ont les pointes de base arrondies et la troisième pointe terminée par un ou plusieurs nœuds. Peut-être le signe de Salomon des Bataks est-il une combinaison du véritable Sigillum Salomonis et du symbole connu de l'éternité: le serpent qui se mord la queue, ou bien, et ceci sous toutes réserves, une transformation du symbole de Yang et de Yin. Ces signes chinois sont les symboles des principes de création masculin et féminin qui, avec les signes du $\mathrm{Pa}$ Kua, les huit trigrammes de la philosophie chinoise, sont apposés par les Chinois sur. les portes de leurs demeures pour protéger les habitants contre les esprits mauvais. Notons que l'on peut accepter comme certain, que vers le commencement de notre ère, des influences culturelles chinoises se sont fait fortement sentir dans les pays montagneux de Sumatra. Pour le Batak, le dessin du tapak Radjah Soeleman doit se faire d'un seul trait. La science magique dit que l'immunité cesse si la figure n'est pas complètement fermée. Les tapak Radjah Soeleman naboroe et dalahi sont dessinés sur des amulettes comme moyen de protection contre les maladies et les malheurs, et aussi tant pour se libérer de l'emprise des diables et des démons que pour leur résister. La base de tout cela est la crainte de l'inconnu et un sentiment de dépendance illimitée envers les puissances surnaturelles.

Le tapak si lima-lima (Sim.

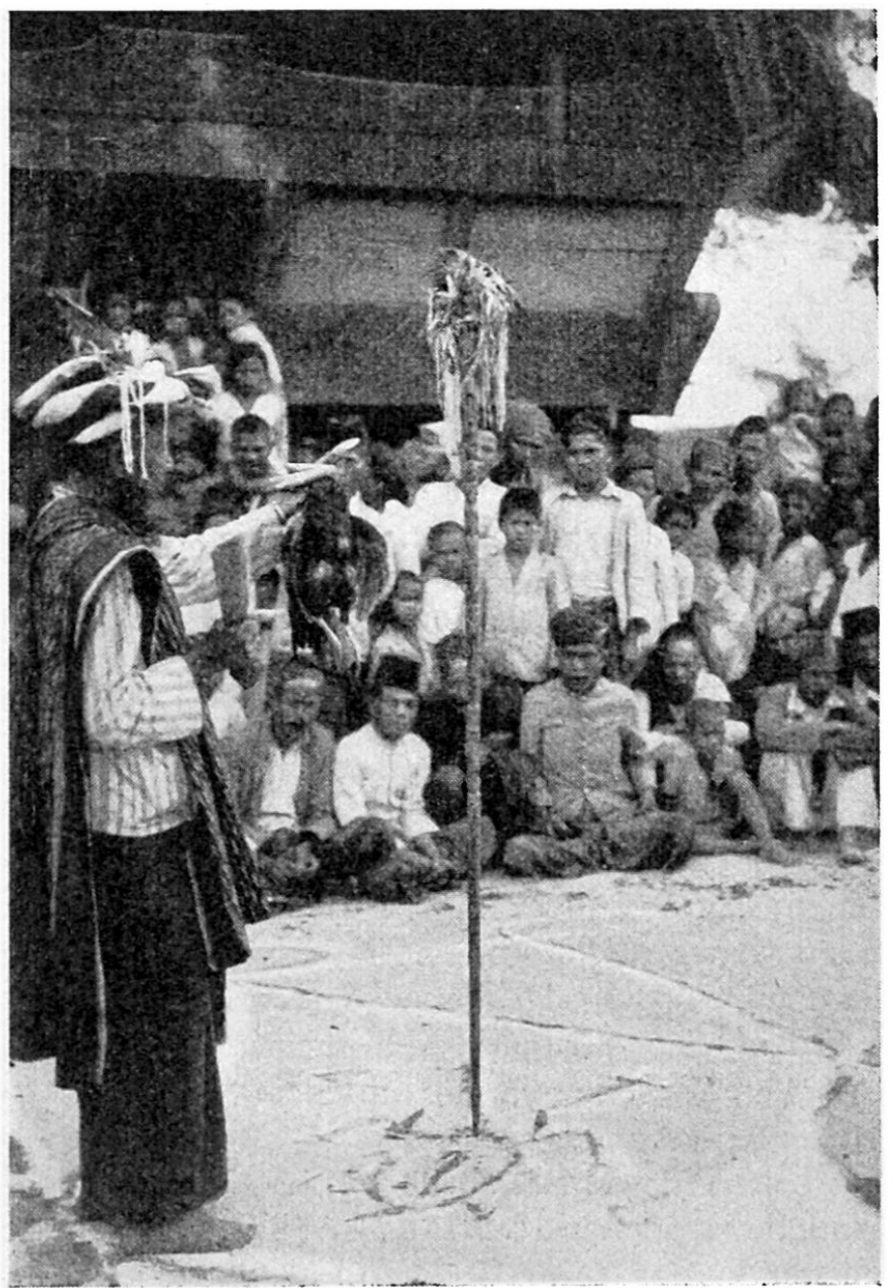

Fig. 2. Prêtre batak (datoe) avec bâton magique pendant une cérémonie, dans laquelle la figure magique du bindoe matogoe joue un rôle. Photo G. L. Tichelman. bat.), fig. 1, VIa, et le tapak si pitoe-pitoe (Sim. bat.), fig. a VI b, les étoiles à cinq ou sept branches, sont des figures magico-mathématiques remontant aux temps les plus reculés. Ce pied de Druide, d'Alpe ou de "Falen», à cinq ou sept pointes, se rencontre dans tous les pays bataks. Le pentagone aux triangles isocèles joints (tapak si lima-lima) était l'insigne des architectes du

4 Dans la magie musulmane, le pentagramme ou l'hexagramme, une seule fois aussi l'heptagramme, comptent comme "sceau de Salomon", dans l'Archipel indonésien, habituellement le pentagramme. Déjà vON BRENNER donna une figure pareille à celle qui est représentée ici sous le nom de «Radjah Suleiman". Il est difficile de déduire cette figure serpentée d'une transformation du pentagramme ou de l'hexagramme, quoique le magie musulmane possède toutes sortes de figures avec des nœuds coulants. Peut-être peut-on voir dans notre figure une reproduction du fouet de Salomon (tjemeti Soelaiman: mal.), tellement redouté des démons? 
moyen âge; il fut déjà en usage chez les gnosticiens et les pythagoriciens, et l'emploi de ce dessin est préhistorique. Le pentagramme occupa toujours une place importante dans la magie des peuples aryens et sémites, et, selon certaines traditions, ce serait cette figure qui était gravée sur la bague du roi Salomon. Comme symbole de l'Harmonie cosmique l'élément central de la liaison avec l'Infini, tant avec la nature qu'avec l'homme, les sectes mystiques moyennâgeuses des Rose-Croisés et les alchimistes employaient aussi l'étoile à six branches, parfois aussi celle à cinq ou sept, selon l'ancienne image de Babylone, soit pour la totalité des planètes, soit pour l'univers. On voit le pentagramme comme motif ornemental à Atjeh (Sumatra) et à Java, par exemple sur la monnaie mystérieuse en usage dans les temples, dont on voit une reproduction dans «Recherches sur les monnaies indigènes de l'Archipel Indonésien et de la Péninsule Malaise» du Professeur H. C. Mirlies (s'Gravenhage, 1871). L'étendard de l'Etat de Madoera, actuellement au Musée de Batavia, montre également un pentagone. Le Batak doit aussi dessiner les pentagrammes et les heptagrammes d'un seul trait. Lorsque la pointe est tournée vers le bas, on les utilise en pratiquant la magie blanche, lorsqu'elle est tournée vers le bas, en exerçant la magie noire.

Dans une "Communication de l'Association des Missions Néerlandaises", de 1902, sous le titre "Le persilihi mbelin", M. Joustra fait mention du toempak salah. Le nom en indique la signification: to empa $\mathrm{k}$ veut dire empêchement, résistance ferme. Son but est de contrer l'influence maligne des esprits. VON BRENNER, qui appelle cette figure "Drudenfuss", reproduit, à côté du pentagone, un heptagone et une figure serpentée, autre que celle dont nous donnons une photo, et qu'il nomme toempak $\mathrm{R}$ adja h Soeleman. Cette figure sert aux mêmes fins que le pentagramme et d'autres signes semblables du moyen âge. Les formules composées des signes $<$ et $\infty$, sept fois répétées dans le soe rat pangoehoem (Sim. bat.), fig. 1 IVa et 1, IVb, et la prescription de droit (nga: ja), représentent des moyens de défense de même que le soerat na so ta roehoem (Sim. bat.), fig. $1 \mathrm{~V}$, qui se compose de sept rangées de caractères bataks que l'on ne peut pas prononcer. On applique ces signes sur des amulettes portées lorsqu'on se met en marche contre l'ennemi (djimat partahanan: Sim. bat. = talisman pour faire obstacle). Le schème de sept joue, comme on le sait, un rôle important dans la cabalistique. Chez les Bataks, on pourrait appeler le sept un nombre sacré. Si l'on répète sept fois le signe nga les mauvais esprits be g oe ne peuvent plus avancer. Ils restent bouche bée et comme décontenancés, fixés sur place. Si l'on écrit sept fois le signe représentant le ja affirmatif, le talisman obtenu est censé rendre dociles et inoffensifs tous les démons. Le s oe rat na so ta róehoe ma pour but de faire naître la confusion et de détruire l'activité des pouvoirs maléfiques.

Le Batak désire entourer de garanties protectrices le village, la communauté à laquelle la porte du village (horbangan: Sim. bat.) donne accès, l'habitation qui doit abriter la famille et la défendre contre les influences nuisibles, visibles ou invisibles venant du dehors, ainsi que la nourriture qui doit, non seulement entretenir la forme tertestre de l'homme, mais aussi rafraîchir et renforcer son âme (tondoei: Sim. bat.; tondi: Toba bat.). Les esprits mauvais invisibles aux profanes, et dont l'homme ne connaît rien, peuvent agir contre lui tant extérieurement, qu'intérieurement et le frapper de toutes les manières. La crainte de ce qui l'entoure entrave totalement la liberté d'action du Batak. Il croit cependant que tout ce qui se trouve à proximité des figures magiques, est à l'abri des influences malignes du dehors. Aussi la gravure sur bois dans les maisons n'est-elle pas purement ornementale, mais a une signification plus étendue: elle sert, en premier lieu, à résister aux pouvoirs et esprits malveillants, à les calmer et à les chasser. Cependant, en interrogeant le Batak, on l'entendra toujours dire que ces figures ne représentent rien et ne sont que des ornements (djagardjagar: Sim. bat.). Les entrées de villages et de demeures sont parmi les premières choses à munir de signes protecteurs contre l'ennemi invisible. La porte du village (horbangan: Sim. bat.), fig. 3, est munie des signes suivants: 

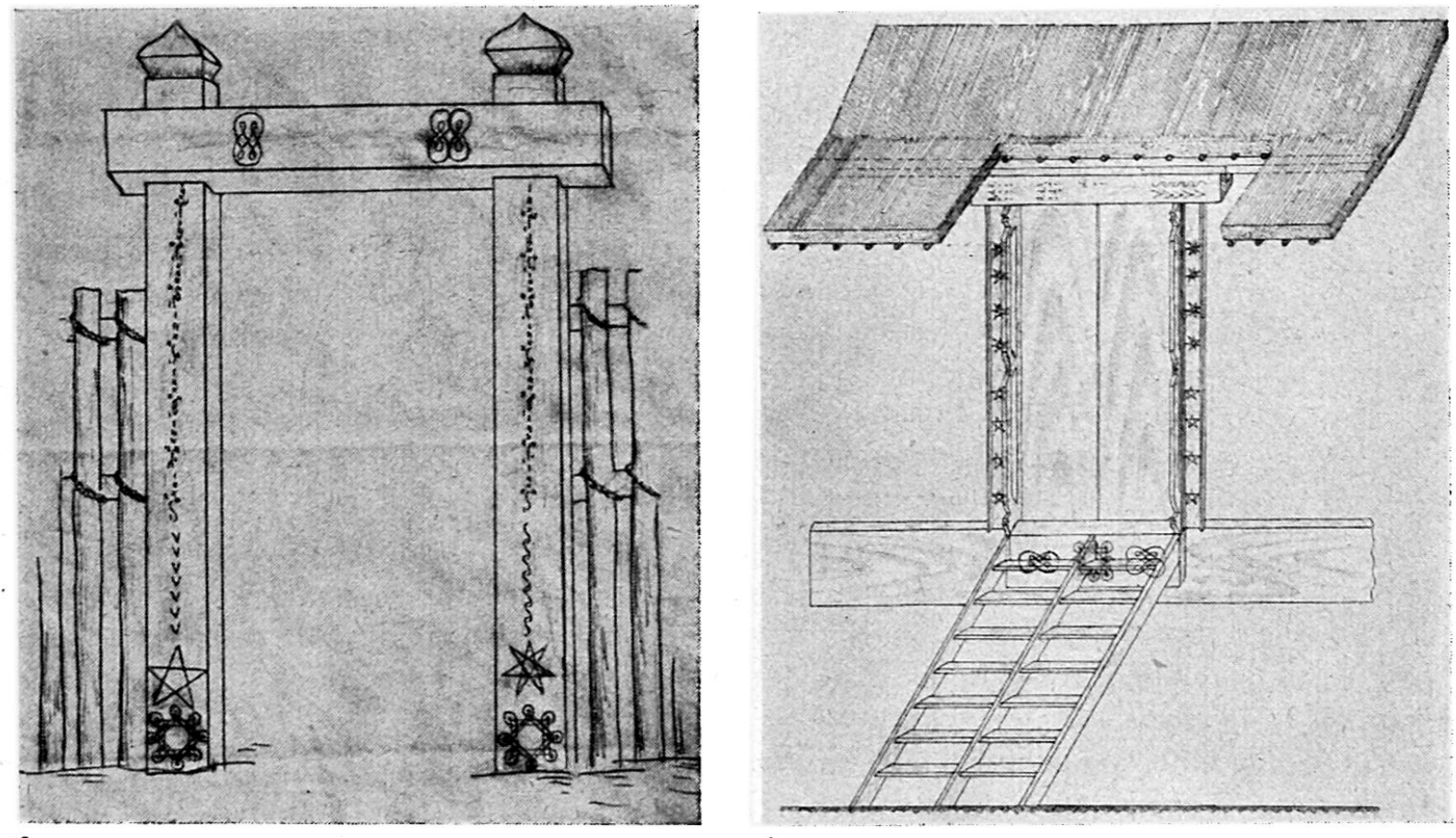

\section{4}

Fig. 3. Porte de village (horbangan) munie de signes magiques protecteurs. Fig. 4. Entrée d'une demeure batake munie des différents signes de défense.

Photos G. L. Tichelman.
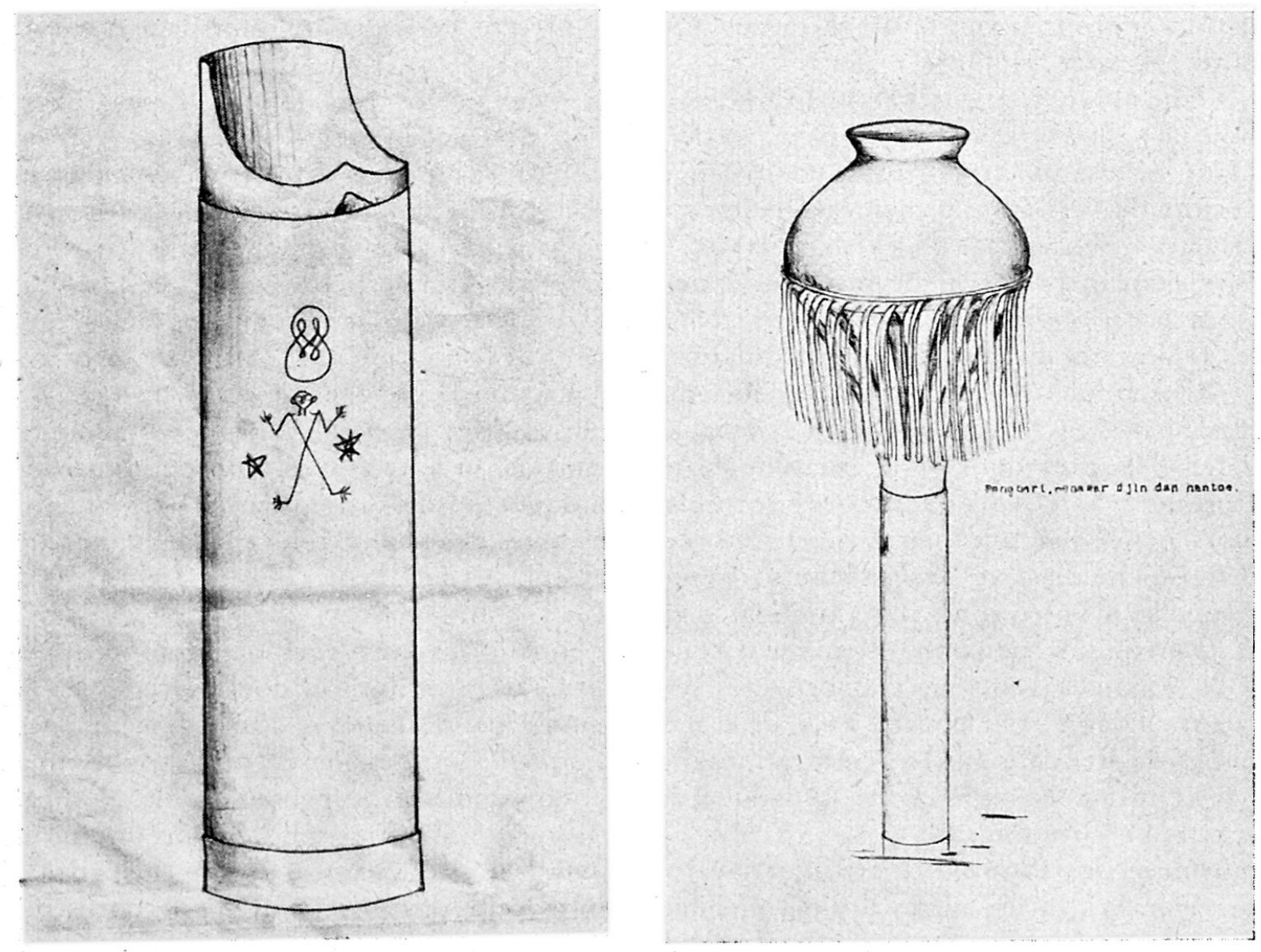

5

6

Fig. 5. Gobelet de bambou nommé panghabahaba et muni de différents signes défensifs (noter le pinarhalak flanqué du pentagramme et de l'heptagramme) servant à attirer la pluie. Fig. 6. Appareil en bambou nommé Panabari et destiné à chasser les mauvais esprits. Photos G. L. Tichelman. 
$1^{\circ}$ Sur la poutre supérieure, on grave le tapak $\mathrm{Radjah}$ Soeleman, le signe masculin et le signe féminin;

$2^{\circ}$ au bas des deux montants de la porte, le bindoe matogoeh;

$3^{\circ}$ au-dessus, sur le montant droit, le tapak si lima-lima;

$4^{\circ}$ sur le montant gauche, le tapak si pitoe-pitoe;

$5^{\circ}$ le soerat pangoehoem avec le signe ja doit être apposé au-dessus de l'heptagramme;

$6^{\circ}$ au-dessus du pentagramme, le soerat pangoehoem, avecle signe ng a atteignant environ la hauteur de l'épaule;

$7^{\circ}$ un peu plus haut, les montants sont munis du soerat na so taroehoem.

L'entrée de la maison (porte de la maison: labah: Sim. bat.), fig. 4, est, elle aussi, protégée par plusieurs signes:

$1^{\circ} \mathrm{Au}$-dessus de la porte, le tapak Radjah Soeleman, masculin et féminin;

$2^{\circ}$ sur le seuil le bindoe matogoeh;

$3^{\circ}$ sur les montants gauche et droit, les mêmes signes que sur les montants de la porte du village. D'autres signes de défense sont gravés ailleurs dans la maison, par exemple au-dessous des fenêtres.

On en dessine également sur la poupée-fétiche nommée parsilihi, parbahbah, satimbang badan ou boeang djangkaö.(Sim. bat.), porsili (Toba bat.). Cette poupée est taillée dans un tronc de bananier (galoeh sitabar), et doit prendre la place d'un malade souffrant d'une maladie chronique. Ces signes sont gravés au couteau sur la poupée, que l'on porte ensuite à la croisée de trois chemins, avec plusieurs plates destinées au sacrifice. Après que les formules magiques d'usage ont été récitées, on dépose le tout au centre du carrefour. C'est de préférence le gendre du malade qui doit faire cela vers minuit.

Une amulette très répandue est la boeloeh pagar (Sim. bat.) faite d'un petit bambou (boeloeh ajam: Sim. bat.), sur lequel on dessine différents signes de défense. Dans cette amulette, on met un mélange (poepoek: Sim. bat.) de feuilles séchées et ensuite pulvérisées des plantes suivantes: salahnipi, tabar-tanar, balik soelpah, silandjoehang, sangkal sipilit et balik hoenda (Sim. bat.). Ce talisman doit spécialement préserver de maladies contagieuses telles que la petite vérole, le choléra, la dysenterie, etc. Les hommes portent cette amulette dans leur ceinture, tandis que les femmes la mettent dans leur foulard de tête (boelangboelang: Sim. bat.).

Une amulette assez semblable, mais qui sert à attirer la pluie, est le panghabahaba (Sim. bat.), fig. 5. On grave au couteau, sur un gobelet de bambou, différents signes défensifs, après quoi on le remplit d'eau, et parfois on le recouvre d'un petit turban. Ensuite le prêtre prononce ses formules magiques au-dessus de l'amulette, qui est alors brisée sur une pierre ou un morceau de bois, également pourvus de signes de défense, tandis que les assistants crient le mot boeroes (voie d'eau). Ce procédé s'appelle manghabahaba (Sim. bat.).

Lorsqu'une personne veut renvoyer à un ennemi les sortilèges que celui-ci lui a jetés, et si elle veut que ces sortilèges se retournent contre lui, on doit procéder de la façon suivante. On dessine avec de la farine sur le sol de l'enclos d'une demeure, les lignes du bindoe matogoeh, à l'intérieur desquelles on dessine un petit bonhomme pinarhalak (Sim. bat.), fig. 2, flanqué de l'heptagramme et du pentagramme, et dont la tête est surmontée du tapak $\mathrm{Radjah}$ Soeleman naboeroe, fig. 5. Puis un prêtre murmure des formules magiques devant un œuf, sur lequel on a dessiné le radjah pinarhalak et les autres figures précitées. Après cela, on pose l'œuf sur le corps du petit bonhomme en le poussant avec une lance (ipantom: Sim. bat.). Si l'œuf se casse, on croit que les puissances malveillantes frapperont l'envoyeur et le rendront malheureux.

S. Liddell Mac Gregor Mathers, dans son livre remarquable "The Key of Solomon the King» (Londres 1909), présente des figures magiques ressemblant au 


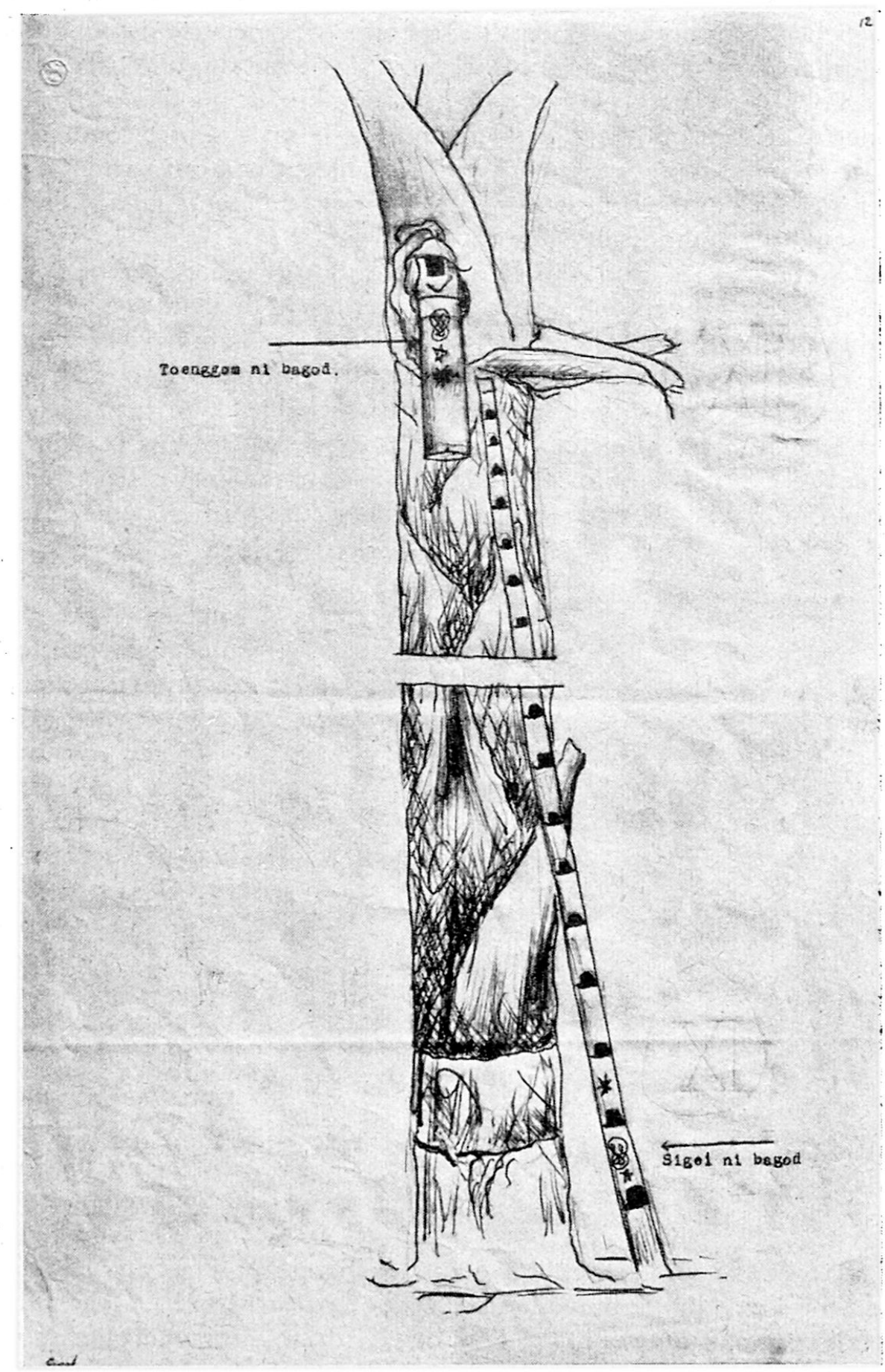

Fig. 7. Récolte de vin de palme. L'échelle et le récipient en bambou sont munis de signes protecteurs. Photo G. L. Trchelman.

bindoeh matogoeh, fig. aA et hA, en mentionnant que celui qui se trouve au dedans de cette figure est protégé comme dans une fortification, contre les pouvoirs malins surnaturels qui l'entourent ${ }^{5}$. Ces deux figures portent le dessin du petit bonhomme pinarhalak, flanqué de l'heptagramme et du pentagramme. Ces signes se retrouvent dans les livres de divination.

Pour lutter contre une maladie (manabari: Sim. bat. et Toba bat.), ou pour prévenir une épidémie, on dessine la même figure, avec de la farine, sur le sol de l'enclos

${ }^{5}$ cf. G. L. TrChelman: «Un des mythes de bâtons magiques des Bataks (Sur la magie des Toba-bataks)» — "Mededeelingen van de Gezaghebbers bij het Binnenlandsch Bestuur in Ned. Indië.» No 39, 1936. 
devant la maison. Mais on procède, ensuite, autrement: au centre de la figure, là où l'on posait l'œuf, on construit un appareil en bambou, de forme singulière, appelé pa nabari (Sim. bat. et Toba bat.), fig. 6. Cet appareil, en combinaison avec les figures dessinées à la farine, détruit le poison des esprits engendrant la maladie, les rend impuissants et tient ces visiteurs indésirables et surnaturels à distance. Ceux qui partent à l'étranger se font dessiner sur les ongles des pouces et des orteils différents signes de défense, qui leur garantissent une bonne santé durant le voyage.

L'échelle confectionnée d'un seul tronc de bambou (sigei: Sim. bat.), par des coupures aux nœuds, très souvent employée pour tirer le vin de palme (bagod: Sim. bat.), est aussi munie à sa base, de plusieurs signes de défense, notamment le ta pa k si limalima, le tapak si pitoe-pitoe et le tapak Radjah Soeleman naboeroe, fig. 7. Pareillement, le gobelet de bambou dans lequel on recueille le vin de palme (toeng gom ni bagod: Sim. bat.) est muni des mêmes signes défensifs, fig. 7. Cela se fait pour empêcher que les esprits des morts (begoe ni namatei: Sim. bat.), ne goûtent en cachette du vin de palme et rendent ainsi malades ceux qui boiraient après.

A une bifurcation (sir-

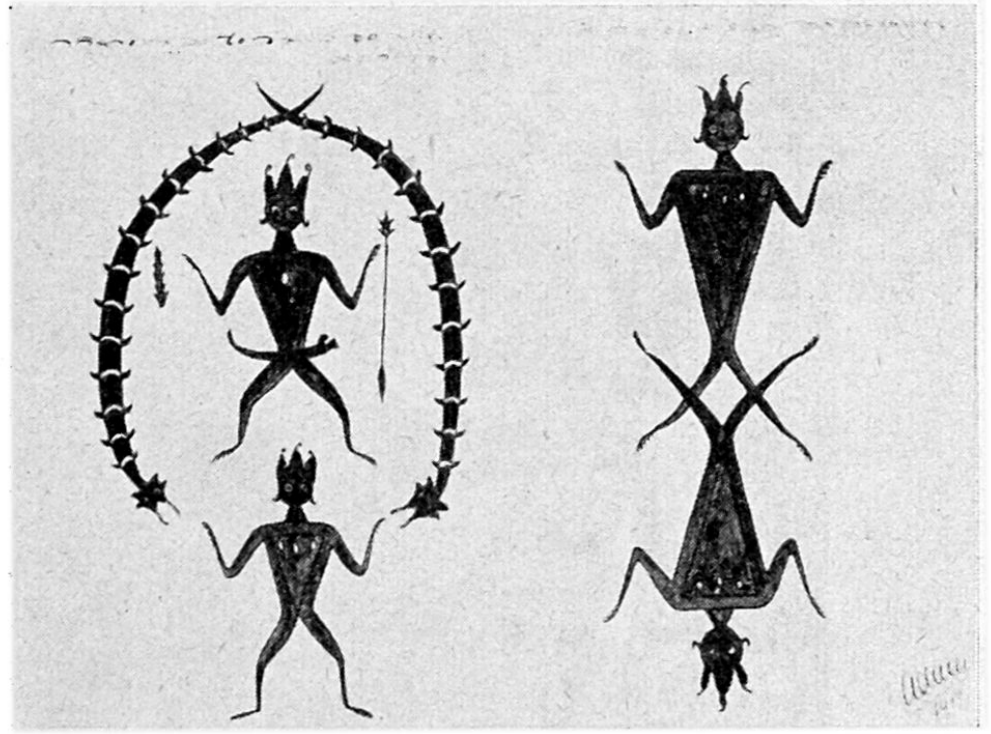

Fig. 8. Dessins magiques tirés d'un livre de divination (poestaha). D'après un dessin de TASsilo Adam. pang: Sim. bat. et Toba bat.) on barre magiquement à l'armée malveillante des esprits, la route qui mène au village (hoe lopah horbou: Sim. bat.), en traçant, au moyen d'un couteau, le tapak Radjah Soeleman naboeroe, le tapak si lima-lima et le tapaksi pitoe-pitoe, dans le sol, au milieu du chemin.

Un certain nombre de figures magiques typiquement batakes, que l'on rencontre à plusieurs reprises dans les livres de divination (poestahas: Sim. bat. et Toba bat.) sont moins connues et moins répandues. Le pin a rhalak tapak radjah, fig. 8 , serait un signe très ancien. Chaque villageois voit dans son chef une personne double: l'être humain, ou l'enveloppe humaine, et l'être quasi-surnaturel, le chef. Il se le représente de ce fait comme étant toujours suivi de ce "double spirituel», marchant dans ses traces. Ceci explique le signe magique, pinarhalak tapak radjah, composé de deux radjah pinarhalaks, attachés par les pieds: le chef et son «double». C'est dans ce double que réside la puissance du chef. Pour le Batak, en effet, la personne du Radjah a une influence protectrice et défensive, grâce à sa force, inspirant la crainte, et à ses propriétés particulières, comparables au mana polynésien et mélanésien. B. J. O. Schrieke, dans son "Livre de Bonang" (note 5, p. 80) insiste particulièrement sur ce point.

Parmi les autres figures et signes magiques, mentionnons encore les suivants, dont certains se rapportent à des végétaux ou à des animaux. Dans la pelure du oette moekkoer (Sim. bat.), en malais linau poeroet, variété de citron et ingrédient courant parmi ceux utilisés lors de presque toutes les cérémonies magiques, on grave, avant l'usage, différents signes de défense. Et particulièrement le radjah ni oette moekkoer (Sim. bat.), qui est un signe spécial, contenant des pouvoirs secrets auxquels on attribue une grande puissance magique. Le roeng padang (Sim. bat.) est 
un moyen magique agissant comme les capitules de la Bardane, une composée. De même que ces capitules s'attachent aux vêtements, le roeng padang s'attache aux esprits malveillants et les gêne dans leurs actions. Pour les animaux citons: les dessins d'une chenille (koeridap: Sim. bat.), d'un scorpion (gansip golang kalaha: Sim. bat., du sanscrit kala), du mille-pattes (lipan: Sim: bat. et Toba bat.), etc. Voici enfin d'autres signes de caractères différents:

Le tapak aledong (Sim. bat.), qui a des propriétés défensives;

le radjah ni hoening (Sim. bat.) - le signe magique "curcuma». Il est considéré comme un gardien infatigable, toujours en mouvement. Il empêche que des fantômes. ou des esprits de défunts (begoes) attaquent quelqu'un au dépourvu;

le bahouta ni hoeta (Sim. bat., du sanscrit bhuta) ou esprit du village;

le sirpang pinarmangmang (Sim. bat.), un "Zellenquadrat» ou "Zellenrechteck», dont WINKLER dit que, tant chez les Grecs qu'en Islam, on lui attribue un pouvoir magique;

le pinongkah ni sirpang (Sim. bat.), talisman pour voyageurs;

le tapak na opat (Sim. bat.) où apparaît le signe nga.

Dans le livre déjà cité de Mathers, on trouve des figures, fig. $\mathrm{nA}$, qui font penser à celles que l'on rencontre dans les livres de divination bataks. De même quelques caractères hébreux, dits "à lunettes», montrent cette similitude de formes.

Les aliments et la boisson sont, pour le Batak, comme pour tous les peuples indonésiens, de la plus haute importance; le repas est toujours un acte très sérieux, et l'on ne peut concevoir de cérémonies magiques sans lui. Il est donc normal que des signes magiques soient incrustés, comme moyens de protection et de sauvegarde, à l'extérieur de la cuillère à riz (sondoek: Sim. bat. et Toba bat.), d'ailleurs faite en bambou. Le soerat pangoehoem (des deux genres) y figure, entouré d'autres signes. Les begoes qui voudraient éventuellement manger le riz et ainsi occasionner des malheurs, en sont empêchés par les différents signes de défense. Le bouchon en bois, fermant la calebasse recouverte de rotin tressé, dans laquelle on conserve l'eau potable (taboetaboe: Sim. bat. et Toba bat.), et que l'on utilise en voyage comme bidon, est, lui aussi, muni de signes protecteurs.

Il est reconnu que le Batak est de caractère plutôt fataliste. Après avoir suivi les prescriptions des prêtres et avoir pratiqué les signes magiques aux endroits désignés, il a la conviction d'avoir fait tout ce qui est humainement possible pour essayer d'échapper au malheur. Il se sent rassuré et laisse le reste au destin.

N. B. Dans cet article, tous les noms indigènes sont écrits selon leur prononciation hollandaise; "oe" correspond done au "ous français. - Abréviations: Sim. bat. = Simaloengoen batak; Toba bat. = Toba batak; Scr. $=$ sanscrit.

\section{ALCUNE FIGURE MAGICHE DEI BATAS E LORO ORIGINE}

L'autore dà una visione generale sui segni magici e sul loro significato nell'attività degli stregoni. Questi segni hanno un duplice valore: da un lato sono semplici segni protettori (con carattere passivo), dall'altro ottengono valore difensivo (con carattere attivo). I sopraddetti segni vengono applicati dai Batas alle case, ai portali e a tutti gli utensili immaginabili ed usati dai sacerdoti nelle loro cerimonie. Per la medesima ragione essi non mancano mai nei libri di magia. L'articolo rappresenta un encomiabile contributo alla conoscenza della pratica della magia presso i Batas.

\section{EINIGE MAGISCHE FIGUREN DER BATAKS UND IHRE HERKUNFT}

Der Verfasser gibt einen Ueberblick über die wichtigsten, zum Teil noch wenig bekannten, in der Wissenschaft und Praxis der Datus (Priester-Medizinmänner) bei den Bataks Sumatras auftretenden magischen Zeichen und Symbole sowie über deren Bedeutung und Verwendung. Diese Zeichen, deren eigenartiger Doppelcharakter sowohl in ihrer mehr passiven Rolle magischer Schutzzeichen als auch in ihrer aktiven Abwehrfunktion zum Ausdruck kommt, werden von den Bataks einerseits an Häusern, Toren und allen möglichen Gebrauchsgegenständen angebracht, doch treten sie andererseits auch im Zuge besonderer, unter der Leitung des Datu stattfindenden Zeremonien auf und fehlen meist in keinem der bekannten batakschen. Zauberbücher, die zu den wichtigsten Requisiten des Priesters gehören. Im einzelnen wird eine Deutung der Herkunft des sehr verbreiteten, unter dem Namen Fußabdruck (tapak) des Königs Salomon dort bekannten Abwehrzeichens in seiner männlichen und weiblichen Ausprägung versucht. 